1 Hierarchical Model for the Role of J-Domain Proteins in Distinct

2

3

4

5

6

7

8

9

10

9

\title{
Cellular Functions
}

Running head: Role of J-domain proteins

Shinya Sugimoto ${ }^{1,2^{*}}$, Kunitoshi Yamanaka ${ }^{3}$, Tatsuya Niwa ${ }^{4}$, Yuki Kinjo ${ }^{1,2}$, Yoshimitsu Mizunoe ${ }^{1,2}$ and Teru Ogura ${ }^{3}$

${ }^{1}$ Department of Bacteriology, The Jikei University School of Medicine, Minato-Ku, Tokyo 105-8461, Japan

${ }^{2}$ Jikei Center for Biofilm Science and Technology, The Jikei University School of Medicine, Minato-Ku, Tokyo 105-8461, Japan

${ }^{3}$ Department of Molecular Cell Biology, Institute of Molecular Embryology and Genetics, Kumamoto University, 2-2-1 Honjo, Chuo-Ku, Kumamoto, 860-0811, Japan

${ }^{4}$ Cell Biology Center, Institute of Innovative Research, Tokyo Institute of Technology, Midori-ku, Yokohama 226-8503, Japan

*Corresponding Author: ssugimoto@jikei.ac.jp (S.S.)

Final Character Count: 25,743 characters (including spaces) 


\section{ABSTRACT}

In Escherichia coli, the major bacterial Hsp70 system consists of DnaK, three J-domain proteins (JDPs: DnaJ, CbpA, and DjIA), and one nucleotide exchange factor (NEF: GrpE). JDPs determine substrate specificity for the Hsp70 system; however, knowledge on their specific role in bacterial cellular functions is limited. In this study, we demonstrated the role of JDPs in bacterial survival during heat stress and the DnaK-regulated formation of curli-extracellular amyloid fibers involved in E. coli biofilm formation. Genetic analysis with a complete set of JDP-null mutant strains demonstrated that only DnaJ is essential for survival at high temperature, while DnaJ and CbpA are indispensable in DnaK regulation of curli production. Additionally, we found that DnaJ and CbpA are involved in the expression of the master regulator CsgD through the folding of MlrA; this keeps CsgA in a translocation-competent state by preventing its aggregation in the cytoplasm. Our findings support a hierarchical model wherein the role of JDPs in the Hsp70 system differs according to individual cellular functions.

Keywords: amyloid/ curli/ Hsp70/ JDPs/ hierarchy

\section{INTRODUCTION}

Proteostasis is the maintenance of protein homeostasis in cells and is essential 
for all life. Key players in proteostasis include molecular chaperones, which assist in protein folding, refolding of denatured and aggregated proteins, protein transport, and quality control of regulatory proteins. Therefore, molecular chaperones are involved in diverse cellular activities including cell division, DNA replication, stress response, organelle functions, and autophagy (Hipp et al., 2019).

The 70-kDa heat shock proteins (Hsp70s) are ubiquitous molecular chaperones involved in a wide variety of cellular functions (Mayer and Kityk, 2015). Hsp70s are ATP-dependent molecular chaperones that consist of an $\mathrm{N}$-terminal nucleotide-binding domain (NBD) and a $25 \mathrm{kDa}$ C-terminal substrate-binding domain (SBD) (Zhu et al., 1996). Hsp70s function via nucleotide-regulated substrate binding and release cycles (Szabo et al., 1994; McCarty et al., 1995). In the ATP-bound state, Hsp70s exhibit low affinity toward substrates; therefore, their rates of substrate binding and release are rapid. In contrast, the ADP-bound state exhibits high substrate affinity, with consequent low rates of substrate binding and release. In the nucleotide-dependent chaperone cycle, Hsp70s require cofactors, known as co-chaperones. Among co-chaperones, J-domain proteins (JDPs), also referred to as Hsp40s, stimulate the ATPase activity of Hsp70s and the binding of substrate proteins (Gässler et al., 1998; Suh et al., 1998, 1999). Conversely, nucleotide exchange factors (NEFs), another type of co-chaperone, induce ADP dissociation from the NBD of Hsp70s and substrate-release from the SBD (Brehmer et al., 2004). Although the amplification and diversification of Hsp70s may be involved in their functional versatility, JDPs far outnumber Hsp70s in the vast majority of life forms 
69 (Kampinga and Craig, 2010), and the multiplicity of JDPs drives the functional diversity of Hsp70s (Craig and Marszalek, 2017). Six JDPs (DnaJ, CbpA, DjIA, $\mathrm{HscB}, \mathrm{Dj} \mathrm{IB}$, and DjIC) have been identified in Escherichia coli, 22 in Saccharomyces cerevisiae, and 41 in humans, whereas there are three Hsp70s in E. coli (DnaK, HscA, and HscC), 16 in S. cerevisiae, and 17 in humans (Table EV1) (Powers and Balch, 2013). In E. coli, three JDPs (DnaJ, CbpA, and DjIA) productively interact with DnaK and play redundant roles in the regulation of DnaK chaperone activity (Sell et al., 1990; Ueguchi et al., 1994; Genevaux et al., 2001; Gur et al., 2004). These observations suggest that DnaJ, CbpA, and DjIA share overlapping functions.

Previously, we demonstrated that DnaK (a bacterial Hsp70) serves an important role in the formation of $E$. coli biofilms-well-organized microbial communities that form on surfaces-and that the production of curli-extracellular functional amyloid fibers—relies on DnaK functions (Arita-Morioka et al., 2015). Curli fibers play crucial roles in biofilm organization and host colonization by adhering to surfaces and holding bacterial cells in a self-produced extracellular matrix (Olsén et al., 1989; Chapman et al., 2002). Secretion and assembly of curli is mediated by a characteristic secretion pathway, known as the nucleation-precipitation mechanism or the type VIII secretion system (Desvaux et al., 2009). In E. coli, seven proteins encoded by two dedicated operons, the curli-specific genes $B A C$ (csgBAC) and DEFG (csgDEFG) operons, are involved in the expression, export, and assembly of the amyloid fibers (Hammar et al., 1995). An alternative sigma factor, RpoS, also known as $\sigma^{S}$ or $\sigma^{38}$, activates expression of the csgDEFG operon (Hammar et al., 
1995; Dudin et al., 2014). In addition, CsgD, the master transcriptional regulator of curli synthesis, directly promotes transcription of the $\operatorname{csgBAC}$ operon (Hammar et al., 1995; Zakikhany et al., 2010). CsgA and CsgB are the major and the minor curli subunits, respectively. Following transport across the cytoplasmic membrane via the Sec translocon, the CsgA and CsgB subunits are exported across the outer membrane in a manner dependent on CsgG, a curli-specific translocation channel (Goyal et al., 2014; Cao et al., 2014). After secretion, CsgB nucleates CsgA subunits into amyloid fibrils (Shu et al., 2012). In addition, CsgE, a periplasmic accessary protein, directs CsgA to CsgG for secretion (Nenninger et al., 2011). CsgF, an extracellular accessory protein, is required for the specific localization and/or nucleation activity of CsgB (Nenninger et al., 2009).

Recently, we found that DnaK multitasks to maintain homeostasis of the key players in curli biogenesis, including regulation of the quantity and de novo folding of RpoS and CsgD, and export of CsgA (Sugimoto et al., 2018); however, curli production was not affected by single knockout of JDPs (DnaJ, CbpA, and DjIA), all of which are known to functionally interact and cooperate with DnaK (Genevaux et al., 2001, 2007). These findings motivated us to investigate whether DnaK works alone or together with specific JDPs in distinct cellular functions, such as curli biogenesis and survival at high temperature. Our results will further our understanding of the role of JDPs and the activity of the DnaK chaperone system.

\section{RESULTS}




\section{Either DnaJ or CbpA is indispensable for curli biogenesis}

118 To address which JDPs are essential for curli biogenesis, we used the Keio 119 collection, a widely used E. coli single-gene knockout library (Baba et al., 2006). We also constructed a complete set of JDP double- and triple-null mutants of the K-12 strain BW25113 (Table EV2) by the one-step method for inactivation of chromosomal genes (Datsenko and Wanner, 2000). Curli production was detected on Congo Red-containing YESCA agar (CR-YESCA: 1\% casamino acids, $0.1 \%$ yeast extract, and $2 \%$ agar) plates at $25^{\circ} \mathrm{C}$ for 2 days. In the strains $\Delta c b p A \Delta d n a J$ and $\Delta c b p A \Delta d j l A \Delta d n a J$, curli production was reduced, as well as in the strains $\Delta d n a K, \Delta \operatorname{csg} A, \Delta \operatorname{csg} D, \Delta \operatorname{csg} G$, and $\Delta r p o S$, while it was not affected in the others (Fig 1A). Curli was also evaluated by immunoblotting for CsgA monomers as described below.

To confirm the responsibility of JDPs in the observed phenotypic changes, we conducted a trans-complementation assay using the JDP-expression plasmids (Table EV2). The plasmids carrying DnaJ and CbpA restored curli production in BW25113 derivatives $\Delta c b p A \Delta d n a J$ (Fig 1B). In contrast, neither DjIA nor DjIA ${ }^{\Delta \mathrm{TM}}$, which lack the transmembrane domain, restored curli production. These results indicate that either DnaJ or $\mathrm{CbpA}$, but not DjIA, is essential for curli production and that DnaJ and CbpA work redundantly in this process.

\section{DnaJ is essential for survival at high temperature}

139 Previously, the requirement for JDPs in the survival of E. coli at high temperature was reported using MC4100 and its isogenic mutants (Sell et al., 1990; Ueguchi 
et al., 1994; Genevaux et al., 2001). These reports showed that deletion of JDPs was not lethal at $30^{\circ} \mathrm{C}$ and that DnaJ, but neither CbpA nor DjIA, was essential for the growth of $\mathrm{MC} 4100$ at $43^{\circ} \mathrm{C}$. We revisited the requirement of JDPs in the survival of BW25113 at high temperature using newly constructed null mutant strains (Table EV2). Our study also revealed that all JDPs were dispensable for growth of BW25113 at $30^{\circ} \mathrm{C}$, and only DnaJ was indispensable for survival at $43^{\circ} \mathrm{C}$ (Fig EV1A). In addition, complementation analysis demonstrated that expression of DnaJ rescued the growth of BW25113 $\Delta d n a J \Delta c b p A$ as well as BW25113 $\Delta d n a J \Delta c b p A \Delta d j I A$ at $43^{\circ} \mathrm{C}$ (Fig $1 \mathrm{C}$ and EV1B). Expression of CbpA or DjIA ${ }^{\Delta T M}$ partially recovered the survival of BW25113 $\Delta d n a J \Delta c b p A$ and $\Delta d n a J$ $\triangle c b p A \triangle d j I A$ at $43^{\circ} \mathrm{C}$ (Fig $1 \mathrm{C}$ and EV1B). These results indicate that DnaJ plays the most pivotal role in survival of $E$. coli at high temperature.

\section{Diversity of DnaK chaperone activities required for distinct phenotypes}

Our results indicate that under physiological conditions, only DnaJ is essential for the growth of E. coli at high temperature, while DnaJ and CbpA work redundantly in curli production. These findings imply the presence of a diversity of DnaK chaperone activities required for different cellular processes. If this is the case, some DnaK mutants with reduced chaperone activity would support curli production but not in survival at high temperature. Here, we focused on a mutant of DnaK with reduced interaction with JDPs. Substitutions of Tyr-145, Asn-147, and Asp-148 to Ala in DnaK (DnaK ${ }^{\mathrm{YND}}$ ) (Fig 2A) caused reduced interaction with DnaJ via its J-domain (Gässler et al., 1998), suggesting that this mutant DnaK interacts very weakly with JDPs. We also focused on defective 
allosteric communication between the NBD and SBD. Previously, two charged residues, a surface-exposed, positively charged residue in the NBD (Lys-155) and a negatively charged residue in the linker connecting NBD and SBD domains (Asp-393), were shown to be important for interdomain communication (Vogel et al., 2006). One of the mutants, DnaK ${ }^{\mathrm{K} 155 \mathrm{D}}$, in which Lys-155 in NBD is replaced with Asp, greatly reduces the stimulation of the substrate dissociation rate by ATP while retaining substrate- and DnaJ-mediated stimulation of ATPase activity (Vogel et al., 2006). In contrast, another mutant, DnaK ${ }^{\mathrm{D} 393 \mathrm{~A}}$, in which Asp-393 is substituted to Ala, drastically reduces the stimulation of substrate dissociation rate by ATP, as well as the substrate- and DnaJ-mediated stimulation of ATPase activity (Vogel et al., 2006).

In our study, expression of these mutant DnaK proteins did not induce recovery from the growth defect of the $\Delta d n a K$ strain at high temperature (Fig $2 \mathrm{~A}$ and B). This indicates that interaction of DnaK with JDPs and allosteric communication between the NBD and SBD of DnaK is indispensable for survival under heat stress conditions, as previously reported (Gässler et al., 1998; Vogel et al., 2006). However, notably, expression of DnaK ${ }^{\mathrm{YND}}$, DnaK ${ }^{\mathrm{K} 155 \mathrm{D}}$, and DnaK $^{\mathrm{D} 393 \mathrm{~A}}$ fully restored curli production in the $\Delta d n a K$ strain (Fig $2 \mathrm{C}$ ). These results support our hypothesis that there is a functional diversity of the DnaK system required for distinct cellular functions.

\section{Loss of DnaJ and CbpA reduces expression of CsgD}

Based on our data, we hypothesized that either DnaJ or CbpA plays an important role for expression and folding of certain proteins associated with curli 
biogenesis. To address this, we investigated the expression levels of several proteins in the BW25113 strains by immunoblotting.

Firstly, we confirmed the expression of chaperone proteins (Fig 3A). As expected, DnaK, DnaJ, and CbpA were not detected in the respective mutant strains, revealing that deletion of these proteins was successfully conducted. Only DjIA was not detected because of the absence of an available specific antibody. The cellular level of DnaK was slightly increased in the strains lacking DnaJ, while that of DnaJ was much higher in the strain $\Delta d n a K$ compared to the other strains. These phenomena can be accounted by the accumulation of $\mathrm{RpoH}$, also known as $\sigma^{32}$ or $\sigma^{\mathrm{H}}$, in these mutant strains, which induces expression of DnaK and DnaJ (Tatsuta et al., 2000). These results are also consistent with the previous observation (Gur et al., 2004). In addition, the cellular level of CbpA in the strain $\Delta r p o S$ was lower than that in the wild-type strain, confirming that the expression of CbpA is positively regulated by RpoS during the stationary growth phase as previously reported (Yamashino et al., 1994).

Secondly, to elucidate the mechanisms of how either DnaJ or CbpA affects the biogenesis of curli, we investigated cellular levels of CsgA, CsgD, and CsgG. In agreement with the results of CR-plate assays (Fig 1A), no CsgA was detected in the strains $\Delta c b p A \Delta d n a J$ and $\triangle c b p A \Delta d j I A \Delta d n a J$ as in the curli-negative strains $\Delta \operatorname{csg} A, \Delta \operatorname{csg} D, \Delta \operatorname{csg} G$, and $\Delta r p o S$ (Fig $3 A$ and B). Likewise, a low level of CsgD was detected in the strains $\triangle c b p A \Delta d n a J$ and $\triangle c b p A \Delta d j I A$ $\Delta d n a J$. In addition, the cellular levels of CsgG in the strains $\triangle c b p A \Delta d n a J$ and $\Delta c b p A \Delta d j l A \Delta d n a J$ were lower than those in the wild-type strain. These data for the strains $\triangle c b p A \Delta d n a J$ and $\triangle c b p A \Delta d j I A \Delta d n a J$ are similar to those of the strain 
$213 \Delta d n a K$. Our previous study indicated that expression of the csgDEFG operon

214 was reduced in the strain $\Delta d n a K$ at the transcription level, which resulted in a

215 reduction in the expression of the $\operatorname{csgBAC}$ operon (Sugimoto et al., 2018).

216 Collectively, these results suggest that reduced expression of csgDEFG operon

217 leads to the decreased expression of $\operatorname{csg} B A C$ operon in the strains $\triangle c b p A$

$218 \Delta$ dnaJ and $\Delta c b p A \Delta d j I A \Delta d n a J$.

219 Next, we analyzed the cellular levels of RpoS, the stationary

220 phase-specific sigma factor, in the BW25113 derivatives as it positively regulates

221 the expression of the csgDEFG operon. A slight reduction in the RpoS level was observed in the strains $\Delta d n a K, \Delta c b p A \Delta d n a J$, and $\Delta c b p A \Delta d j I A \Delta d n a J$ (Fig 3).

The decreased level of RpoS in the strain $\Delta d n a K$ may be due to accelerated

these results, the cellular activity of RpoS through the measurement of catalase

activity was reduced in these mutant strains (Fig EV2), suggesting that a partially

reduced RpoS level resulted in a decrease in the expression of the csgDEFG

operon. However, the activity of RpoS in the strains $\Delta d n a K, \Delta c b p A \Delta d n a J$, and

$\triangle c b p A \Delta d j l A \Delta d n a J$ was not low enough to shut the expression of the $c s g D E F G$

operon down. Therefore, other mechanisms may account for the drastic

reduction of the cellular CsgD level in the strain $\triangle c b p A \Delta d n a J$ and $\Delta c b p A \Delta d j l A$

$\Delta d n a J$.

Either DnaJ or CbpA is involved in folding of transcriptional regulator MIrA

To investigate whether either DnaJ or CbpA is important for the expression of 
vitro. MlrA was synthesized using an in vitro translation system (PURE System; Shimizu et al., 2001), in the presence or absence of complete and incomplete sets of DnaK/DnaJ/GrpE (KJE) or DnaK/CbpA/GrpE (KAE). De novo synthesized MlrA readily formed aggregates and a complete set of KJE or KAE assisted the folding of MlrA (Fig 4A and EV3A). Incomplete sets of DnaK/DnaJ (KJ), DnaJ/GrpE (JE) and DnaJ alone (J) also promoted the solubility of MIrA, indicating that the folding of MlrA strongly relied on DnaJ. It should be noted that CbpA can compensate for DnaJ in KJE-assisted folding of MlrA (Fig 4A and EV3A). In contrast, DnaK alone (K), DnaK/CbpA (KA), DnaK/GrpE (KE), $\operatorname{CbpA} / \operatorname{GrpE}(\mathrm{AE}), \mathrm{CbpA}$ alone (A), and GrpE (E) showed no or only slight stimulation of the solubility of MlrA (Fig 4A and EV3A). These results indicate that either DnaJ or CbpA is required for the DnaK chaperone system to efficiently fold MlrA. In addition, these findings are in good agreement with those already described, indicating that either DnaJ or CbpA is indispensable for efficient curli production (Fig 1-3).

Next, we examined whether de novo folding of CsgD required either DnaJ or CbpA, because its folding is assisted by the complete DnaK chaperone system (KJE) (Sugimoto et al., 2018). Our study revealed that CsgD formed aggregates in the absence of the chaperones and that KJE and KAE promoted CsgD folding (Fig 4B and EV3B). In contrast, DnaK alone did not support CsgD folding under the tested conditions (Fig 4B and EV3B). Interestingly, incomplete sets of the DnaK systems (KJ and KE) moderately enhanced the solubility of CsgD in vitro (Fig 4B and EV3B). DnaJ, CbpA, GrpE, and combinations of DnaJ and GrpE or CbpA and GrpE did not assist the folding of CsgD (Fig 4B and 
261 EV3B). These results indicate that these incomplete DnaK systems (KJ and KE) can act as molecular chaperones and that JDPs are dispensable at least for the folding of CsgD.

\section{Either DnaJ or CbpA is required for maintenance of CsgA in a}

\section{translocation-competent state}

267 Transport of CsgA across the cytoplasmic membrane is pivotal for curli biogenesis. In this process, DnaK acts on the transport precursor of CsgA to maintain its transport competent state via direct interaction with its $\mathrm{N}$-terminal aggregation-prone signal peptide (Sugimoto et al., 2018). Firstly, we examined whether co-expression of CsgBAEFG was able to complement the defect of curli production in the strain BW2513 $\Delta d n a J \Delta c b p A$, in which CsgA was not detected, as shown in Fig 3. Previously, we showed that introduction of the plasmid pCsgBAEFG was able to recover the production of curli in the BW25113 strains $\Delta \operatorname{csg} A, \Delta \operatorname{csg} B, \Delta \operatorname{csg} E, \Delta \operatorname{csg} \mathrm{F}$, and $\Delta \operatorname{csg} G$, confirming the plasmid was functional (Sugimoto et al., 2018). However, expression of CsgBAEFG did not restore the production of curli in $\Delta d n a J \Delta c b p A(F i g)$ A), suggesting that secretion of CsgA was defective at the step of translocation from the cytoplasm to the periplasm or from the periplasm to the extracellular milieu. protein in the BW25113 parental strain and its isogenic $\Delta d n a K$ strain.

284 Subsequently, we observed that CsgA-sfGFP localized at the periplasm of the 
parental strain, whereas it formed aggregates in the cytoplasm of the $\Delta d n a K$ mutant (Sugimoto et al., 2018). In this study, we expressed the fusion protein in BW25113 derivative strains $\Delta d n a J, \Delta c b p A$, and $\Delta d n a J \Delta c b p A$. Fluorescence of sfGFP was observed at the periphery of the single null strains $\Delta d n a J$ and $\Delta c b p A$, indicating that CsgA-sfGFP was translocated to the periplasm. In contrast, it formed aggregates in the cytoplasm of the double knockout strain $\triangle d n a J \Delta c b p A$ (Fig 5B). These results suggest the requirement of either DnaJ or CbpA for translocation of CsgA from the cytoplasm to the periplasm.

Taken together, our results indicate that either DnaJ or CbpA is required for DnaK to assist the translocation of CsgA to the periplasm during curli biogenesis.

\section{DISCUSSION}

Previously, we showed that DnaK is essential for curli biogenesis and biofilm formation via the maintenance of certain important proteins including RpoS, CsgD, and CsgA (Sugimoto et al., 2018). Here, we demonstrated that DnaJ and CbpA were essential for this process, while DjIA was not essential and that DnaJ and $\mathrm{CbpA}$ work redundantly in this process (Fig 1-3 and EV1). DnaK cooperates with DnaJ to assist in protein folding and to repair damaged proteins under harmful conditions, such as high temperature at $43^{\circ} \mathrm{C}$, which causes denaturation and aggregation of numerous proteins (Fig 1C and EV1). The DnaK system efficiently prevents the aggregation of diverse thermolabile proteins, including $150-200$ species, corresponding to $15-25 \%$ of detected proteins, under physiological heat stress conditions (Mogk et al., 1999). In 

addition, the disaggregation activities of the DnaK/CbpA/GrpE and DnaK/DjIA/GrpE systems were lower than that of the DnaK/DnaJ/GrpE system (Gur et al. 2004), suggesting that only DnaJ is essential for survival under severe stress conditions (e.g., heat stress at $\geq 43^{\circ} \mathrm{C}$ ). Our model is consistent with the current body of research in several respects: (1) In curli biogenesis, only a subset of proteins (minimally RpoS, CsgD, and CsgA) require DnaK for their correct folding or export (Sugimoto et al., 2018). Our study also suggests that efficient folding of MlrA needs the DnaK system including DnaK, GrpE, and either DnaJ or CbpA (Fig 3-5). (2) DnaK mutants (DnaK ${ }^{\mathrm{YND}}$, DnaK ${ }^{\mathrm{K} 155 \mathrm{D}}$, and $\mathrm{DnaK}^{\mathrm{D} 393 \mathrm{~A}}$ ) with reduced basal activity were functional in the curli production but not in survival at high temperature of $43^{\circ} \mathrm{C}$ (Fig 2). (3) E. coli produces curli during the stationary phase of growth, in which expression of CbpA is induced via RpoS (Fig $3 A$ ). Therefore, the contribution of CbpA to the promotion of the DnaK function may be emphasized during curli biogenesis. This growth phase-specific selection of JDPs is a reasonable strategy for improving bacterial survival and fitness under nutrient-depleted conditions (e.g., during stationary phase) and adaptation during host colonization. (4) DjIA is an inner membrane anchoring JDP in E. coli (Clarke et al., 1996). Therefore, DjlA may be involved in the quality control of membrane proteins and trafficking of exported proteins (Kelley and Georgopoulos, 1997). However, DjlA is dispensable for maintenance of proteins associated with curli production.

Based on these observations, we propose a hierarchical model whereby Hsp70 chaperone activities regulate proteostasis in distinct cellular functions (Fig 6). When a large amount/variety of proteins in the cell are injured by severe 
stress conditions (e.g., heat stress at $\geq 43^{\circ} \mathrm{C}$ ), full specification of the DnaK system (DnaK/DnaJ/GrpE) prevents their aggregation and the repair of toxic protein aggregates. In contrast, under certain conditions (e.g., curli biogenesis), a moderately active DnaK system (DnaK/CbpA/GrpE) fulfills its chaperone function by handling only a subset of proteins. Although DnaK/DjIA/GrpE and DnaK alone did not support the production of curli, they may function weakly by holding substrate proteins (Evans et al., 2011), which might be associated with a specific phenotype such as colanic acid production (Kelley and Georgopoulos, 1997) and other unknown cellular functions. This model is also supported by the results of DnaK mutants in which DnaK ${ }^{\mathrm{YND}}$, DnaK ${ }^{\mathrm{K} 155 \mathrm{D}}$, and DnaK ${ }^{\mathrm{D} 393 \mathrm{~A}}$ restored the curli production in the $\Delta d n a K$ strain despite the failure to support the growth at high temperature (Fig 2). In addition, neither DnaK ${ }^{\mathrm{K} 70 \mathrm{~A}}$ which possesses a defective ATPase activity nor DnaK ${ }^{\mathrm{V} 436 \mathrm{~F}}$ which retains decreased substrate affinity was able to rescue the thermosensitivity as well as the deficiency in curli production of the $\Delta d n a K$ strain (Sugimoto et al., 2018).

This study focused on the requirement of JDPs in the production of bacterial amyloid fibers. In addition to JDPs, DnaK cooperates with GrpE, a well-conserved bacterial NEF. We were also interested in the requirement of GrpE in curli biogenesis. However, deletion of the grpE gene from the genome of BW25113 was difficult because of its lethality in E. coli (Ang and Georgopoulos, 1989). Genetic analysis using an available grpE null strain derived from E. coli strain C600 (Ang and Georgopoulos, 1989; Sugimoto et al., 2008) suggested that GrpE may be dispensable for curli production (Sugimoto et al., unpublished). Further careful study is needed to clarify this observation. 

proteostasis. It has been previously suggested that Hsp70 chaperone systems co-evolved with the proteome to regulate the physiological state of the cell (Powers and Balch, 2013). The number of Hsp70s increases roughly linearly as the size of the genome increases (Powers and Balch, 2013). In addition, the number of JDPs is often much higher than that of Hsp70s in almost all life forms (Kampinga and Craig, 2010). Moreover, in contrast to Hsp70s, JDPs show a greater degree of sequence and structural divergence. These insights imply that they may play a major role in driving the multi-functionality of $\mathrm{Hsp} 70$ chaperone systems (Craig and Marszalek, 2017). In contrast, the single set of

DnaK-DnaJ-GrpE is well conserved in Gram-positive bacteria, such as bacteria. Therefore, smaller numbers of Hsp70s and JDPs in Gram-positive bacteria may be anticipated, as the proteome size is also small, minimizing the work of the Hsp70 system. In the cases of microorganisms with extremely small genomes, such as Candidatus Hodgkinia cicadicola and Candidatus Carsonella ruddii, only either DnaJ or GrpE is present, and there is a single Hsp70/DnaK (Table EV1). How do such incomplete DnaK systems (so called proto-DnaK systems) work in maintaining proteostasis? These organisms possess quite small numbers of proteins; therefore, the roles of the DnaK systems must be minimal. In this situation, activity of proto-DnaK systems (DnaK/DnaJ and DnaK/GrpE) may be sufficient for regulating a limited proteome to aid survival of these microorganisms (Fig 6). This notion is consistent with our results that 
381

382

383

384

385

386

387

388

389

390

391

392

393

394

395

396

397

398

399

400

401

402

403

404

incomplete sets of the E. coli DnaK chaperone system (DnaK/DnaJ and

DnaK/GrpE) can contribute to folding of certain proteins (e.g., MlrA and CsgD)

(Fig 4B and EV3B). Whether other cellular processes require either

full-specification DnaK systems or only proto-DnaK systems remains unclear; some proteins may require DnaK and either DnaJ or GrpE for their folding. It is expected that proteins with lower affinity with DnaK need only DnaJ, since DnaK alone is not able to capture them and these proteins can be released spontaneously from DnaK despite the absence of GrpE. In contrast, folding of proteins with higher affinity with DnaK may depend on GrpE because, although DnaK alone is able to bind them, the release of proteins tightly bound to DnaK requires GrpE. The lower affinity proteins may be expressed predominantly in Candidatus bacteria that possess the DnaK/DnaJ system, whereas the higher affinity proteins may be expressed in Candidatus bacteria that possess the DnaK-/GrpE system. These insights into diversification and evolution of the Hsp70 chaperone system, in combination with our data, imply that primitive organisms may use proto-DnaK systems to manage their small proteomes.

\section{METHODS AND MATERIALS}

\section{Bacterial strains}

The E. coli strains used in this study are listed in Table EV2. All strains were cultivated in LB medium ( $1 \%$ tryptone, $0.5 \%$ yeast extract, $0.5 \% \mathrm{NaCl}$ ) or YESCA medium ( $1 \%$ casamino acid, $0.1 \%$ yeast extract). When appropriate, the medium was supplemented with $30 \mu \mathrm{g} / \mathrm{ml}$ chloramphenicol, $50 \mu \mathrm{g} / \mathrm{ml}$ kanamycin, or $100 \mu \mathrm{g} / \mathrm{ml}$ ampicillin. 
Construction of $E$. coli null mutant strains

407

The JDP-null mutant strains of BW25113 (Table EV2) were constructed by the one-step method for inactivation of chromosomal genes (Datsenko and Wanner, 2000; Baba et al., 2006). The plasmids and primers used for gene knockout are listed in Tables EV2 and EV3, respectively.

\section{Construction of plasmids}

413 The ASKA clone plasmids (pASKA-DnaJ, pASKA-CbpA, pASKA-DjIA, pASKA-CsgD, and pASKA-MIrA) were provided by the National Institute of Genetics (Shizuoka, Japan). For construction of pDjIA ${ }^{\Delta T M}$ (Table EV2), the DNA encoding the transmembrane domain was deleted by inverse PCR using KOD Plus Neo DNA polymerase (Toyobo, Osaka, Japan), pASKA-DjIA as a template, and a primer set DjIA-deltaTM-F and DjIA-deltaTM-R (Table EV3). $\left(D_{n a K}{ }^{\mathrm{YND}}, \mathrm{DnaK}^{\mathrm{K} 155 \mathrm{D}}\right.$, and DnaK $\left.{ }^{\mathrm{D} 393 \mathrm{~A}}\right)$, site-directed mutagenesis was performed by inverse PCR using KOD Plus Neo DNA polymerase (Toyobo, Osaka, Japan), pDnaK ${ }^{\mathrm{WT}}$ as a template, and the following primer sets: dnaK-YND-F/dnaK-YND-R, dnaK-K155D-F/dnaK-K155D-R, and dnaK-D393A-F/dnaK-D393A-R. The resultant plasmids were termed $\mathrm{pDnaK}^{\mathrm{YND}}$, pDnaK ${ }^{\mathrm{K} 155 \mathrm{D}}$, and $\mathrm{pDnaK}^{\mathrm{D} 393 \mathrm{~A}}$, respectively (Table EV2).

The plasmids were analyzed by DNA sequencing (Eurofins Genomics, Tokyo, Japan). Primers used in this study were synthesized by Thermo Fisher 
and are summarized in Table EV3.

430

\section{Protein purification}

432

Recombinant DnaK, DnaJ, and GrpE were purified as described previously

(Niwa et al., 2012). CbpA was expressed in E. coli BL21(DE3). Cells harboring pCU60 were grown at $30^{\circ} \mathrm{C}$ in $2 \times \mathrm{YT}$ medium containing $100 \mu \mathrm{g} / \mathrm{ml}$ ampicillin, and expression of CbpA was induced by adding IPTG (1 $\mathrm{mM})$ and incubating at $30^{\circ} \mathrm{C}$ for $3 \mathrm{~h}$. Cells from 2-L culture were harvested by centrifugation and resuspended in $50 \mathrm{ml}$ buffer $\mathrm{A}[10 \mathrm{mM}$ Tris- $\mathrm{HCl}(\mathrm{pH} 8.0), 1 \mathrm{mM}$ DTT, 10\% glycerol] supplemented with a protease inhibitor cocktail. After sonication on ice, cell lysates were centrifuged at $12,000 \times g$ for 60 min at $4^{\circ} \mathrm{C}$, and the supernatant was loaded onto a 5-ml bed volume of HiTrap Heparin column (GE Healthcare, Pittsburgh, PA, USA) pre-equilibrated with buffer A. CbpA was eluted using a 0 $1,000 \mathrm{mM} \mathrm{NaCl}$ gradient in buffer A. Each fraction containing CbpA was pooled and further purified by chromatography using a HiTrap Q column (GE Healthcare) and a 0-1,000 mM NaCl gradient in buffer A. Purified CbpA was confirmed by LC-MS/MS and quantified using a Bradford Assay Kit.

\section{Antibodies}

448 Rabbit anti-DnaJ and rabbit anti-RpoH antisera were gifted by Dr. B. Bukau 449 (Gamer et al., 1992). The other antibodies were prepared as previously reported 450 (Arita-Morioka et al., 2018; Sugimoto et al., 2018). 
453 Curli formation was assayed at $25^{\circ} \mathrm{C}$ on CR-containing YESCA ( $1 \%$ casamino 454 acid, $0.1 \%$ yeast extract, $2 \%$ agar) plates as previously reported (Arita-Morioka et al., 2018; Sugimoto et al., 2018). When needed, $30 \mu \mathrm{g} / \mathrm{ml}$ chloramphenicol was added to supplement select transformants.

\section{Thermosensitivity assay}

E. coli BW25113 derivative cells were grown at $30^{\circ} \mathrm{C}$ in LB medium overnight. Overnight cultures were serially diluted 10 -fold, and $5 \mu$ of these dilutions were spotted onto LB agar plates. If required, $30 \mu \mathrm{g} / \mathrm{ml}$ chloramphenicol was added to supplement select transformants. These plates were incubated at $30^{\circ} \mathrm{C}$ or $43^{\circ} \mathrm{C}$ for $24 \mathrm{~h}$.

\section{Immunoblotting}

For detection of CsgA, CsgD, CsgG, RpoS, RpoD, RpoH, DnaK, DnaJ, CbpA, and His-tagged MlrA, immunoblotting was performed as previously reported (Arita-Morioka et al., 2018; Sugimoto et al., 2018). After SDS-PAGE, proteins were transferred to polyvinylidene difluoride membranes using the iBlot 2 dry blotting system (Thermo Fisher) following the manufacturer's instructions.

471 Membranes were blocked with blocking solution [1-5\% skimmed milk,

472 Tris-buffered saline containing $0.1 \%(\mathrm{v} / \mathrm{v})$ Tween $20($ TBS-T)] for at least $1 \mathrm{~h}$ at $25^{\circ} \mathrm{C}$ or overnight at $4^{\circ} \mathrm{C}$. After gentle washing with TBS-T, the membrane was

474 incubated with appropriate primary antibodies for at least $1 \mathrm{~h}$ at $25^{\circ} \mathrm{C}$ or overnight at $4^{\circ} \mathrm{C}$. Membranes were subsequently probed with appropriate 
477 washing the membrane three times with TBS-T, signals were detected using the

478 ECL Prime Western Blotting Detection Reagent (GE Healthcare) and an

479 ImageQuant LAS-4000 system (GE Healthcare). When required, signal

480 intensities were quantified with ImageQuant TL software version 7.0 (GE

481 Healthcare).

Primary antibodies were diluted into CanGet Signal 1 (Toyobo) as

follows: anti-CsgA (1/1,000), anti-CsgD (1/200), anti-CsgG (1/5,000-1/1,000),

anti-RpoS (1/10,000-1/1,000), anti-RpoD (1/10,000-1/1,000), anti-RpoH

$(1 / 2,000)$, anti-DnaK (1/10,000), anti-DnaJ (1/10,000), anti-CbpA $(1 / 10,000)$, and

anti-His (1/10,000). HRP-conjugated goat anti-rabbit IgG and HRP-conjugated

487

goat anti-mouse IgG secondary antibodies were diluted 1/50,000 and 1/10,000,

respectively, in CanGet Signal 2 (Toyobo).

For detection of CsgA monomers, curli fibers were depolymerized into

subunits by treatment with hexafluoroisopropanol (HFIP) before SDS-PAGE

(Sugimoto et al. 2018). Bacterial cells (1 mg) were suspended in $10 \mu \mathrm{STTE}$

buffer [10 mM Tris-HCl (pH 8.0), 100 mM NaCl, 2 mM EDTA] and mixed well with

$50 \mu \mathrm{l} \mathrm{HFIP.} \mathrm{After} \mathrm{brief} \mathrm{sonication,} \mathrm{samples} \mathrm{were} \mathrm{vacuum} \mathrm{dried} \mathrm{using} \mathrm{a} \mathrm{SpeedVac}$

vacuum concentrator (Thermo Fisher) at $45^{\circ} \mathrm{C}$ for more than $30 \mathrm{~min}$. The

HFIP-treatment was repeated. The dried materials were dissolved in $40 \mu \mathrm{l} 8 \mathrm{M}$

urea solution. After brief sonication in a water bath for $5 \mathrm{~min}$ at room temperature,

497 the solutions were mixed with an equal volume of $2 \times$ SDS sample buffer [150

498 mM Tris- $\mathrm{HCl}$ (pH 6.8), 4\% SDS, 20\% glycerol, 10\% 2-mercaptoethanol]. 
501

502

503

504

505

506

507

508

509

510

511

512

513

514

515

516

517

518

519

520

521

522

523

524

\section{In vitro protein folding assay}

De novo folding of CsgD and MlrA was analyzed using the PURE System (Shimizu et al., 2001) as previously reported (Niwa et al., 2012; Sugimoto et al., 2018). The $\operatorname{csg} D$ gene was amplified from the CsgD-expression plasmid pASKA-CsgD (Table EV2) by PCR using KOD Plus DNA polymerase v. 2 (Toyobo) and the primer set Pure-Niwa-F and Pure-CsgD-R (Table EV3). The mIrA gene was amplified from the MlrA-expression plasmid pASKA-MIrA (Table EV2) by PCR using Phusion High-Fidelity DNA polymerase (New England Biolabs, Tokyo, Japan) and the primer set Pure-Niwa-F and Pure-Niwa-R (Table EV3). The amplified DNA fragments were purified and incubated with PUREfrex solution (GeneFrontier Corp., Chiba, Japan) at $37^{\circ} \mathrm{C}$ for $3-4 \mathrm{~h}$ according to the manufacturer's instructions. When required, reaction mixtures $(20-40 \mu \mathrm{l})$ were supplemented with DnaK $(5 \mu \mathrm{M})$, DnaJ $(1 \mu \mathrm{M}), \operatorname{CbpA}(1 \mu \mathrm{M})$, and/or GrpE $(1 \mu \mathrm{M})$. After incubation, aliquots $(10-20 \mu \mathrm{l})$ of the solution were obtained as the total fractions and were centrifuged at $20,000 \times g$ for $30 \mathrm{~min}$ at $4^{\circ} \mathrm{C}$ to separate the soluble and insoluble fractions. The equivalent volumes of the total, soluble, and insoluble fractions were mixed with $2 \times$ SDS sample buffer. After boiling at $95^{\circ} \mathrm{C}$ for $5 \mathrm{~min}$, proteins were resolved by $15 \%$ SDS-PAGE and stained with CBB. For detection of CsgD and MlrA, immunoblotting was performed as described above.

\section{Fluorescence microscopy}

Transport and aggregation of CsgA-sfGFP was observed in E. coli as previously reported (Sugimoto et al., 2018) with a slight modification. E. coli expressing CsgA-sfGFP were grown in LB medium supplemented with $100 \mu \mathrm{g} / \mathrm{ml}$ ampicillin 
at $30^{\circ} \mathrm{C}$ overnight. A small aliquot of the overnight cultures was placed on a slide and covered with a coverslip. Fluorescence of sfGFP was observed under a fluorescence microscope (Nikon, Tokyo, Japan) equipped with B2 (excitation filter, 450-490 nm; barrier filter, $520 \mathrm{~nm}$ ) and G2A (excitation filter, 510-560 nm; barrier filter, $590 \mathrm{~nm}$ ) filters. In this study, no arabinose was supplemented into the media, because leaky expression from the plasmid was sufficient to visualize fluorescence.

\section{Statistical analysis}

One-way ANOVA with Dunnett's post hoc test was used to determine whether any of the groups exhibited a statistically significant difference in the solubility of MIrA and CsgD analyzed by the PURE System. All experiments were performed at least three times. For all analyses, $P<0.05$ was considered statistically significant. ACKNOWLEDGEMENTS

543 We acknowledge Dr. B. Bukau for providing the anti-DnaJ and anti-RpoH antibodies, Dr. Y. Ueguchi for gifting pCU60, Dr. N. Tani for LC-MS/MS analysis,

545 National BioResource Project (NBRP, Japan) for the Keio collection and ASKA

546 clone. We also thank A. Terao, N. Toda, N. Fukuda, D. Fujioka, and H. Iso for 547 experimental assistance and other members in Department of Bacteriology, Jikei 548 University School of Medicine for stimulating discussions. We are grateful to Dr. 
T. Kanamori for supporting the analysis using the PURE System.

Joint International Research [Fostering Joint International Research (A)] to S.S. from JSPS (18KK0429), a grant to Y.M. from the MEXT-Supported Program for the Strategic Research Foundation at Private Universities, 2012-2016, the Joint

Usage/Research Center for Developmental Medicine, IMEG, Kumamoto

556

University, The Uehara Memorial Foundation to S.S., and by JST ERATO Grant

Number JPMJER1502.

558

559

\section{AUTHOR CONTRIBUTIONS}

560

S.S., K.Y., and T.O. planned the project. S.S. designed the experiments and experiments and analyzed the data. Y.K. and Y.M. supported the project. S.S. and K.Y. wrote the paper with input from all co-authors.

\section{CONFLICT OF INTEREST}

There is no conflict of interest.

\section{REFERENCES}

Ang, D., and Georgopoulos, C. (1989). The heat-shock-regulated grpE gene of Escherichia coli is required for bacterial growth at all temperatures but is

571 dispensable in certain mutant backgrounds. J. Bacteriol. 171, 2748-2755. 
573 Arita-Morioka, K., Yamanaka, K., Mizunoe, Y., Ogura, T., and Sugimoto, S. 574 (2015). Novel strategy for biofilm inhibition by using small molecules targeting 575 molecular chaperone DnaK. Antimicrob. Agents Chemother. 59, 633-641.

576

577 Arita-Morioka, K.I., Yamanaka, K., Mizunoe, Y., Tanaka, Y., Ogura, T., and 578 Sugimoto, S. (2018). Inhibitory effects of Myricetin derivatives on 579 curli-dependent biofilm formation in Escherichia coli. Sci. Rep. 8, 8452.

580

581

Baba, T., Ara, T., Hasegawa, M., Takai, Y., Okumura, Y., Baba, M., Datsenko, 582 K.A., Tomita, M., Wanner, B.L., and Mori, H. (2006). Construction of Escherichia coli K-12 in-frame, single-gene knockout mutants: the Keio collection. Mol. Syst. Biol. 2, 2006.0008.

585

586

Brehmer, D., Gässler, C., Rist, W., Mayer, M.P., and Bukau, B. (2004). Influence of GrpE on DnaK-substrate interactions. J. Biol. Chem. 279, 27957-27964.

589 Brown, P.K., Dozois, C.M., Nickerson, C.A., Zuppardo, A., Terlonge, J., and 590 Curtiss, R. 3rd. (2001). MlrA, a novel regulator of curli (AgF) and extracellular matrix synthesis by Escherichia coli and Salmonella enterica serovar Typhimurium. Mol. Microbiol. 41, 349-363.

594 Cao, B., Zhao, Y., Kou, Y., Ni, D., Zhang, X.C., and Huang, Y. (2014). Structure 595 of the nonameric bacterial amyloid secretion channel. Proc. Natl. Acad. Sci. USA 596 111, E5439-5444. 
598 Chapman, M.R., Robinson, L.S., Pinkner, J.S., Roth, R., Heuser, J., Hammar, M., 599 Normark, S., and Hultgren, S.J. (2002). Role of Escherichia coli curli operons in 600 directing amyloid fiber formation. Science 295, 851-855.

601

602 Clarke, D.J., Jacq, A., and Holland, I.B. (1996). A novel DnaJ-like protein in 603 Escherichia coli inserts into the cytoplasmic membrane with a type III topology. 604 Mol. Microbiol. 20, 1273-1286.

605

606 Craig, E.A., and Marszalek, J. (2017). How do J-proteins get Hsp70 to do so 607 many different things? Trends Biochem. Sci. 42, 355-368.

608

609 Datsenko, K.A., and Wanner, B.L. (2000). One-step inactivation of chromosomal 610 genes in Escherichia coli K-12 using PCR products. Proc. Natl. Acad. Sci. USA $61197,6640-6645$.

612

613 Desvaux, M., Hébraud, M., Talon, R., and Henderson, I.R. (2009). Secretion and 614 subcellular localizations of bacterial proteins: a semantic awareness issue. 615 Trends Microbiol. 17, 139-145.

616

617 Dudin, O., Geiselmann, J., Ogasawara, H., Ishihama, A., and Lacour, S. (2014). 618 Repression of flagellar genes in exponential phase by CsgD and CpxR, two 619 crucial modulators of Escherichia coli biofilm formation. J. Bacteriol. 196, $620 \quad 707-715$. 
622 Evans, M.L., Schmidt, J.C., Ilbert, M., Doyle, S.M., Quan, S., Bardwell, J.C., 623 Jakob, U., Wickner, S., and Chapman, M.R. (2011). E. coli chaperones DnaK, 624 Hsp33 and Spy inhibit bacterial functional amyloid assembly. Prion 5, 323-334.

625

626 Gamer, J., Bujard, H., and Bukau, B. (1992). Physical interaction between heat 627 shock proteins DnaK, DnaJ, and GrpE and the bacterial heat shock transcription 628 factor sigma 32. Cell 69, 833-842.

629

630 Gässler, C.S., Buchberger, A., Laufen, T., Mayer, M.P., Schröder, H., Valencia, A., 631 and Bukau, B. (1998). Mutations in the DnaK chaperone affecting interaction with the DnaJ cochaperone. Proc. Natl. Acad. Sci. USA 95, 15229-15234.

633

634 Genevaux, P., Schwager, F., Georgopoulos, C., and Kelley, W.L. (2001). The djIA 635 gene acts synergistically with dnaJ in promoting Escherichia coli growth. J. 636 Bacteriol. 183, 5747-5750.

637

638 Genevaux, P., Georgopoulos, C., and Kelley, W.L. (2007). The Hsp70 chaperone 639 machines of Escherichia coli: a paradigm for the repartition of chaperone 640 functions. Mol. Microbiol. 66, 840-857.

641

642 Goyal, P., Krasteva, P.V., Van Gerven, N., Gubellini, F., Van den Broeck, I., 643 Troupiotis-Tsaïlaki, A., Jonckheere, W., Péhau-Arnaudet, G., Pinkner, J.S., 644 Chapman, M.R., et al. (2014). Structural and mechanistic insights into the 
bacterial amyloid secretion channel CsgG. Nature 516, 250-253.

646

647 Gur, E., Biran, D., Shechter, N., Genevaux, P., Georgopoulos, C., and Ron, E.Z. 648 (2004). The Escherichia coli DjlA and CbpA proteins can substitute for DnaJ in 649 DnaK-mediated protein disaggregation. J. Bacteriol. 186, 7236-7242.

650

651 Hammar, M., Arnqvist, A., Bian, Z., Olsén, A., and Normark, S. (1995).

652 Expression of two csg operons is required for production of fibronectin- and 653 congo red-binding curli polymers in Escherichia coli K-12. Mol. Microbiol. 18, 654 661-670.

655

656

Hipp, M.S., Kasturi, P., and Hartl, F.U. (2019). The proteostasis network and its

657 decline in ageing. Nat. Rev. Mol. Cell. Biol. 20, 421-435.

658

659

Kampinga, H.H., and Craig, E.A. (2010). The HSP70 chaperone machinery: J proteins as drivers of functional specificity. Nat. Rev. Mol. Cell Biol. 11, 579-592.

661

662 Kelley, W.L., and Georgopoulos, C. (1997). Positive control of the

663 two-component RcsC/B signal transduction network by DjIA: a member of the

664 DnaJ family of molecular chaperones in Escherichia coli. Mol. Microbiol. 25, 665 913-931.

666

667 Kitagawa, M., Ara, T., Arifuzzaman, M., loka-Nakamichi, T., Inamoto, E., 668 Toyonaga, H., and Mori, H. (2005). Complete set of ORF clones of Escherichia 
coli ASKA library (a complete set of E. coli K-12 ORF archive): unique resources

671

Mayer, M.P., and Kityk, R. (2015). Insights into the molecular mechanism of allostery in Hsp70s. Front. Mol. Biosci. 2, 58.

674

McCarty, J.S., Buchberger, A., Reinstein, J., and Bukau, B. (1995). The role of

ATP in the functional cycle of the DnaK chaperone system. J. Mol. Biol. 249,

678

679

Mogk, A., Tomoyasu, T., Goloubinoff, P., Rüdiger, S., Röder, D., Langen, H., and

680

Bukau, B. (1999). Identification of thermolabile Escherichia coli proteins:

681

prevention and reversion of aggregation by DnaK and CIpB. EMBO J. 18,

682 6934-6949.

683

684

Nenninger, A.A., Robinson, L.S., and Hultgren, S.J. (2009). Localized and efficient curli nucleation requires the chaperone-like amyloid assembly protein CsgF. Proc. Natl. Acad. Sci. USA 106, 900-905.

687

688 Nenninger, A.A., Robinson, L.S., Hammer, N.D., Epstein, E.A., Badtke, M.P., 689 Hultgren, S.J., and Chapman, M.R. (2011). CsgE is a curli secretion specificity 690 factor that prevents amyloid fibre aggregation. Mol. Microbiol. 81, 486-499.

692 Niwa, T., Kanamori, T., Ueda, T., and Taguchi, H. (2012). Global analysis of 
chaperone effects using a reconstituted cell-free translation system. Proc. Natl.

694

Acad. Sci. USA 109, 8937-8942.

695

696

Olsén, A., Jonsson, A., and Normark, S. (1989). Fibronectin binding mediated by

a novel class of surface organelles on Escherichia coli. Nature 338, 652-655.

698

699

Powers, E.T., and Balch, W.E. (2013). Diversity in the origins of proteostasis

networks - a driver for protein function in evolution. Nat. Rev. Mol. Cell Biol. 14,

701

237-248.

702

703

Rockabrand, D., Livers, K., Austin, T., Kaiser, R., Jensen, D., Burgess, R., and

704

Blum, P. (1998). Roles of DnaK and RpoS in starvation-induced thermotolerance

705

of Escherichia coli. J. Bacteriol. 180, 846-854.

706

707

Sell, S.M., Eisen, C., Ang, D., Zylicz, M., and Georgopoulos, C. (1990). Isolation

708

and characterization of dnaJ null mutants of Escherichia coli. J. Bacteriol. 172,

4827-4835.

710

711 Shimizu, Y., Inoue, A., Tomari, Y., Suzuki, T., Yokogawa, T., Nishikawa, K., and

Ueda T. (2001). Cell-free translation reconstituted with purified components. Nat.

713

Biotechnol. 19, 751-755.

714

715 Shu, Q., Crick, S.L., Pinkner, J.S., Ford, B., Hultgren, S.J., and Frieden, C.

716 (2012). The E. coli CsgB nucleator of curli assembles to $\beta$-sheet oligomers that 
717 alter the CsgA fibrillization mechanism. Proc. Natl. Acad. Sci. USA 109,

$718 \quad 6502-6507$.

719

720 Suh, W.C., Burkholder, W.F., Lu, C.Z., Zhao, X., Gottesman, M.E., and Gross,

721 C.A. (1998). Interaction of the Hsp70 molecular chaperone, DnaK, with its

722 cochaperone DnaJ. Proc. Natl. Acad. Sci. USA 95, 15223-15228.

723

724 Sugimoto, S., Saruwatari, K., Higashi, C., and Sonomoto, K. (2008). The proper

725 ratio of GrpE to DnaK is important for protein quality control by the

726

DnaK-DnaJ-GrpE chaperone system and for cell division. Microbiology 154,

727

1876-1885.

728

729 Sugimoto, S., Arita-Morioka, K.I., Terao, A., Yamanaka, K., Ogura, T., and

730 Mizunoe, Y. (2018). Multitasking of Hsp70 chaperone in the biogenesis of

731 bacterial functional amyloids. Commun. Biol. 1, 52.

732

733 Suh, W.C., Lu, C.Z., and Gross, C.A. (1999). Structural features required for the

734 interaction of the Hsp70 molecular chaperone DnaK with its cochaperone DnaJ.

735 J. Biol. Chem. 274, 30534-30539.

736

737 Szabo, A., Langer, T., Schröder, H., Flanagan, J., Bukau, B., and Hartl, F.U.

738 (1994). The ATP hydrolysis-dependent reaction cycle of the Escherichia coli

739 Hsp70 system DnaK, DnaJ, and GrpE. Proc. Natl. Acad. Sci. USA

740

22,10345-10349. 
742 Tatsuta, T., Joob, D.M., Calendar, R., Akiyama, Y., and Ogura, T. (2000).

743 Evidence for an active role of the DnaK chaperone system in the degradation of 744 sigma(32). FEBS Lett. 478, 271-275.

745

746 Ueguchi, C., Kakeda, M., Yamada, H., and Mizuno, T. (1994). An analogue of the

747 DnaJ molecular chaperone in Escherichia coli. Proc. Natl. Acad. Sci. USA 91, $748 \quad 1054-1058$.

Vogel, M., Mayer, M.P., and Bukau B. (2006). Allosteric regulation of Hsp70 chaperones involves a conserved interdomain linker. J. Biol. Chem. 281,

752 $38705-38711$.

753

754

Warnecke, T. (2012). Loss of the DnaK-DnaJ-GrpE chaperone system among 755 the Aquificales. Mol. Biol. Evol. 29, 3485-3495.

756

757 Yamashino, T., Kakeda, M., Ueguchi, C., Mizuno, T. (1994). An analogue of the

758 DnaJ molecular chaperone whose expression is controlled by sigma s during the 759 stationary phase and phosphate starvation in Escherichia coli. Mol. Microbiol. 13, 760 475-483.

761

762 Zakikhany, K., Harrington, C.R., Nimtz, M., Hinton, J.C., and Römling, U. (2010).

763 Unphosphorylated CsgD controls biofilm formation in Salmonella enterica

764 serovar Typhimurium. Mol. Microbiol. 77, 771-786. 
Zhu, X., Zhao, X., Burkholder, W.F., Gragerov, A., Ogata, C.M., Gottesman, M.E., and Hendrickson, W.A. (1996). Structural analysis of substrate binding by the molecular chaperone DnaK. Science 272, 1606-1614.

FIGURE LEGENDS

Figure 1. Effects of JDP deletion on curli biogenesis

(A) Curli production in E. coli BW25113 and its isogenic mutants were examined on Congo Red (CR)-containing YESCA plates (CR-YESCA). Strains were grown at $25^{\circ} \mathrm{C}$ for 2 days. The strains $\Delta \operatorname{csg} A, \Delta \operatorname{csg} D, \Delta c s g D$, and $\Delta r p o S$ were used as negative controls. (B) Curli production in BW25113 and its isogenic $\Delta c b p A$ $\Delta d n a J$ strains transformed with pCA24N (empty vector) and the indicated JDP-expression plasmids were examined on CR-YESCA plates supplemented with chloramphenicol. Strains were grown at $25^{\circ} \mathrm{C}$ for 2 days. (C) Thermosensitivity of the indicated strains was assayed on LB agar plates supplemented with chloramphenicol. Ten-fold serial dilutions of the overnight cultures were spotted on the plates. Plates were incubated at $30^{\circ} \mathrm{C}$ or $43^{\circ} \mathrm{C}$ for $24 \mathrm{~h}$.

Figure 2. Complementation of the dnaK-null strain with defective DnaK mutants in cooperation with JDPs and interdomain communication

(A) The domain structure of DnaK and mutants used in this study. Arrowheads represent mutation sites. (B) The thermosensitivity of the indicated strains was assayed on LB agar plates supplemented with chloramphenicol. Ten-fold serial 
789

790

791

792

793

794

795

796

797

798

799

800

801

802

803

804

805

806

807

808

809

810

811

812

dilutions of the overnight cultures were spotted on the plates. Plates were incubated at $30^{\circ} \mathrm{C}$ or $43^{\circ} \mathrm{C}$ for $24 \mathrm{~h}$. (C) Curli production in the strains was examined on CR-YESCA plates supplemented with chloramphenicol. The plates were incubated at $25^{\circ} \mathrm{C}$ for 2 days.

\section{Figure 3. Effects of JDPs on expression of curli-related proteins}

(A) BW25113 and its isogenic null mutants were grown on YESCA plates at $25^{\circ} \mathrm{C}$ for 2 days. Expression of curli-related proteins, chaperones, and sigma factors was analyzed by immunoblotting. CsgA monomers were depolymerized with hexafluoroisopropanol (HFIP). All experiments were conducted using total protein samples. Relative protein levels of CsgA, CsgD, CsgG, and RpoS were quantified based on the band intensity of immunoblots. All experiments were repeated at least three times to ensure accuracy and averaged values with standard deviations were calculated. The band intensities in the BW25113 parental strain were defined as $100 \%$.

Figure 4. Effects of complete and incomplete DnaK chaperone systems on de novo folding of transcriptional regulators

(A, B) De novo folding of MlrA and CsgD was analyzed in the absence (Control) or presence of the indicated chaperone proteins using a cell-free translation system (PURE System). The solubilities (\%) of MlrA (A) and CsgD (B) were calculated based on the band intensity of immunoblots. All experiments were repeated at least three times to ensure accuracy and averaged values with standard deviations were calculated. K, DnaK; KJ, DnaK/DnaJ; KA, DnaK/CbpA; 
813 KE, DnaK/GrpE; KJE, DnaK/DnaJ/GrpE; KAE, DnaK/CbpA/GrpE; JE,

814 DnaJ/GrpE; AE, CbpA/GrpE; J, DnaJ; A, CbpA; E, GrpE. ***, P $<0.001 .{ }^{*}, \mathrm{P}<$ 815 0.05. NS, not significant.

816

817 Figure 5. Effects of DnaJ and CbpA on translocation of CsgA-sfGFP

818 (A) Curli production in the indicated strains was examined on CR-YESCA plates 819 supplemented with chloramphenicol as described in Fig 1B. The plates were 820 incubated at $25^{\circ} \mathrm{C}$ for 2 days. (B) Translocation of CsgA across the cytoplasmic membrane was analyzed in BW25113 derivatives using a CsgA-sfGFP fusion protein (Sugimoto et al., 2018). E. coli cells were grown in LB medium supplemented with $100 \mu \mathrm{g} / \mathrm{ml}$ ampicillin. Scales, $10 \mu \mathrm{m}$.

824

825

Figure 6. A hierarchical model of DnaK chaperone activities

826

The proposed model for DnaK chaperone activities showing that the most powerful DnaK/DnaJ/GrpE system is essential for survival under severe stress conditions (e.g., high temperature) for dealing with a wide variety of substrate proteins. The middle active DnaK/CbpA/GrpE system (Gur et al. 2004) can perform specific cellular functions (e.g., curli biogenesis). The modest active DnaK/DjIA/GrpE system is not able to support curli production but may work for unknown cellular activities. DnaK alone engages in holding substrate proteins, but its cellular functions remain elusive. Respective DnaK mutants with hierarchical activities are indicated based on the results presented in this study and our previous one (Sugimoto et al., 2018). 
EXPANDED VIEW LEGENDS

838

Figure EV1. Effects of JDP deletion on thermotolerance of E. coli strain BW25113

(A) Thermosensitivity of the indicated strains was assayed on LB agar plates.

Ten-fold serial dilutions of the overnight cultures were spotted on the plates and incubated at $30^{\circ} \mathrm{C}$ or $43^{\circ} \mathrm{C}$ for $24 \mathrm{~h}$. (B) BW25113 and its isogenic $\Delta c b p A \Delta d n a J$ $\triangle d j l A$ strains were transformed with pCA24N (empty vector) or the indicated JDP-expression plasmids. The strains were grown at $30^{\circ} \mathrm{C}$ or $43^{\circ} \mathrm{C}$ for $24 \mathrm{~h}$ on LB agar plates supplemented with chloramphenicol.

Figure EV2. Activity of RpoS in BW25113 derivative strains

Relative RpoS activity was assayed by measuring catalase activity as previously reported (Sugimoto et al., 2018). All experiments were repeated at least three times to ensure accuracy and averaged values with standard deviations were calculated. The activity of the BW25113 parental strain was defined as $100 \%$.

Figure EV3. Effects of complete and incomplete DnaK chaperone systems on de novo folding of transcriptional regulators MIrA and CsgD (A, B) De novo folding of MlrA and CsgD was analyzed in the absence (Control) or presence of the indicated chaperone proteins using a cell-free translation system (PURE System). His-tagged MlrA (A) and CsgD (B) were detected using anti-His antibody and anti-CsgD antibody, respectively. All experiments were repeated at least three times to ensure accuracy and representative images are shown. K, DnaK; KJ, DnaK/DnaJ; KA, DnaK/CbpA; KE, DnaK/GrpE; KJE, 
bioRxiv preprint doi: https://doi.org/10.1101/2020.06.12.147645; this version posted June 12, 2020. The copyright holder for this preprint (which was not certified by peer review) is the author/funder. All rights reserved. No reuse allowed without permission.

861 DnaK/DnaJ/GrpE; KAE, DnaK/CbpA/GrpE; JE, DnaJ/GrpE; AE, CbpA/GrpE; J,

862 DnaJ; A, CbpA; E, GrpE. 


\section{Sugimoto et al. Figure 1}

A

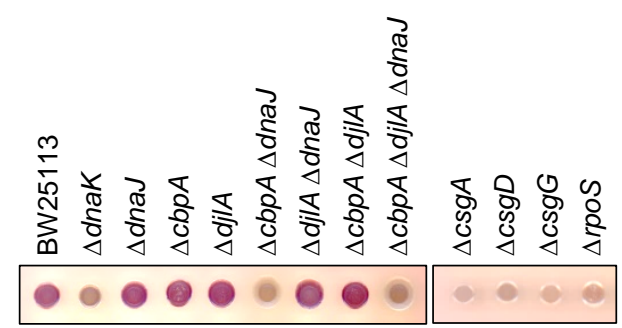

B

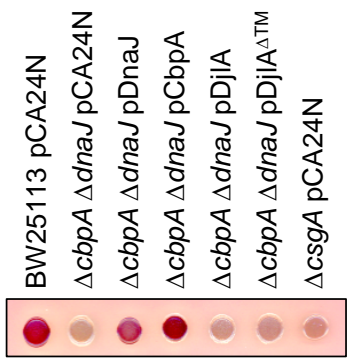

C

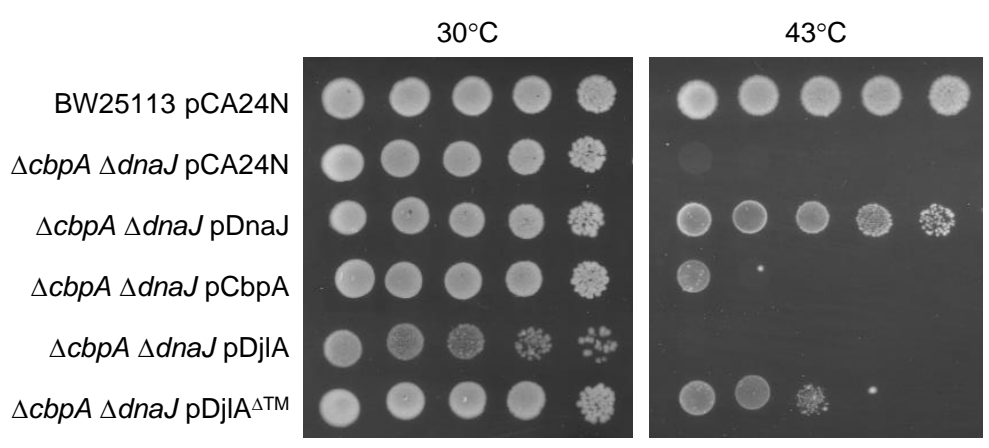




\section{Sugimoto et al. Figure 2}

A

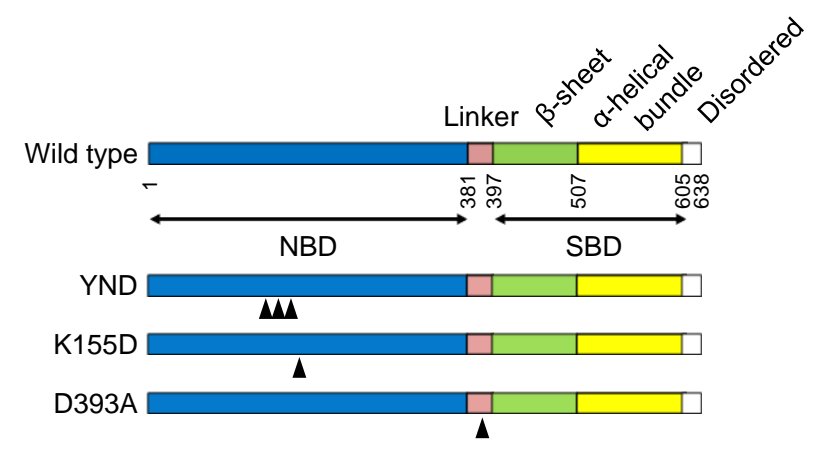

B

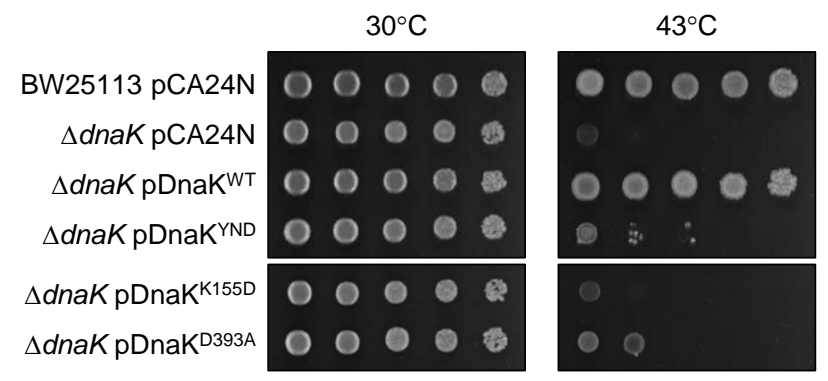

C

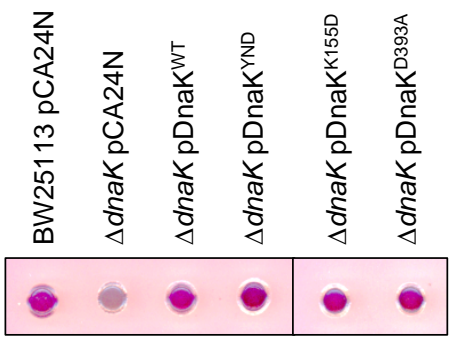




\section{Sugimoto et al. Figure 3}

A

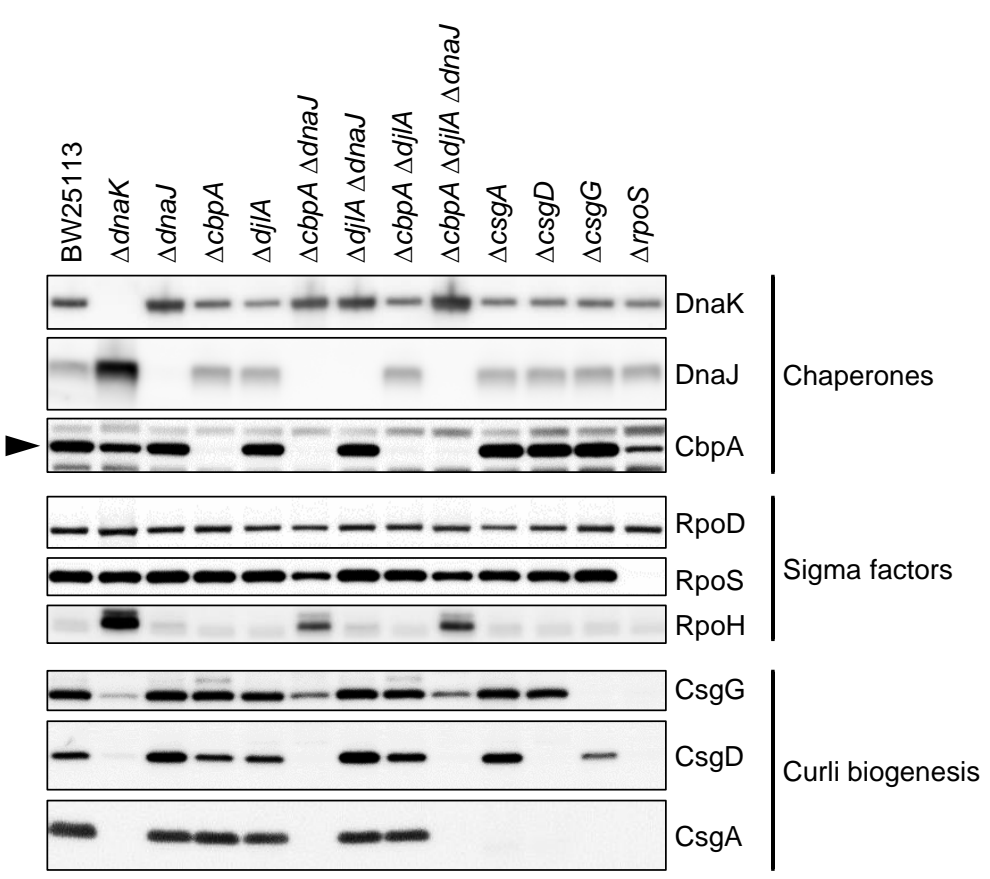

B
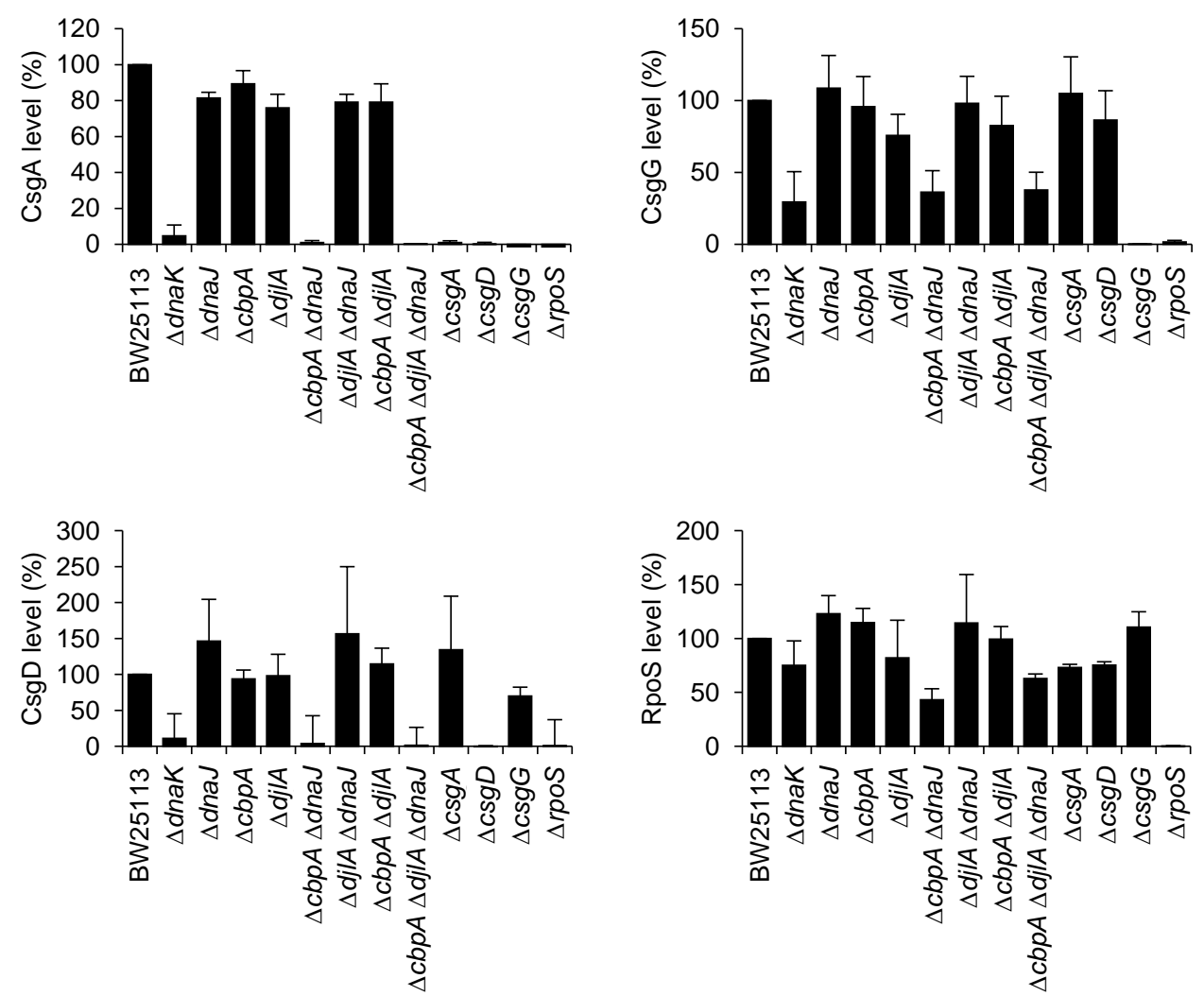


\section{Sugimoto et al. Figure 4}

A

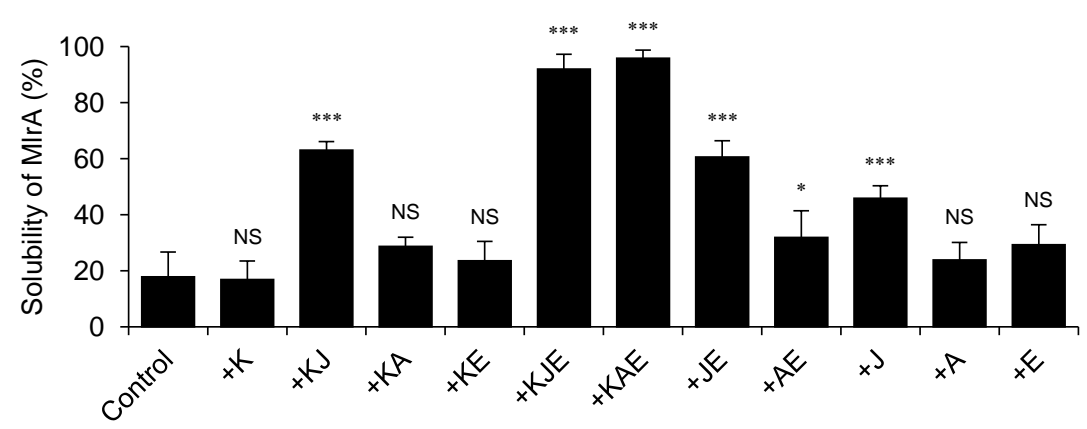

B

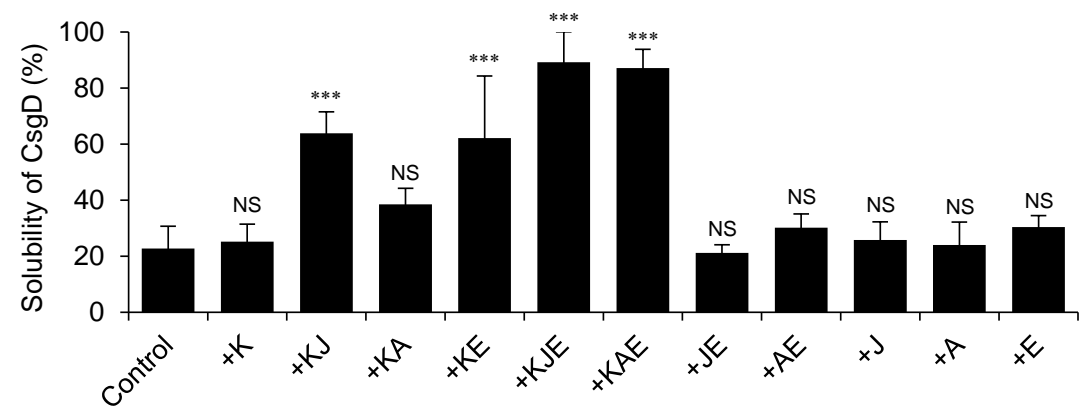


Sugimoto et al. Figure 5

A

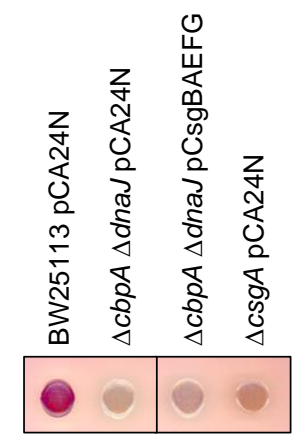

B

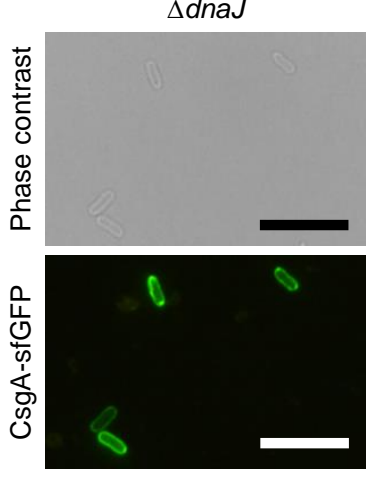

$\triangle c b p A$

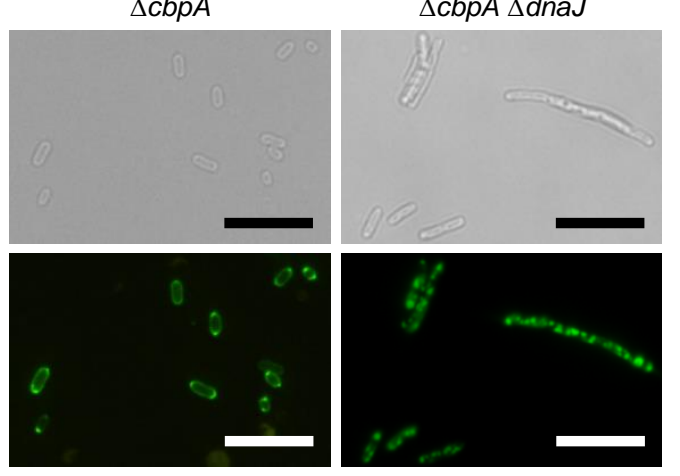




\section{Sugimoto et al. Figure 6}
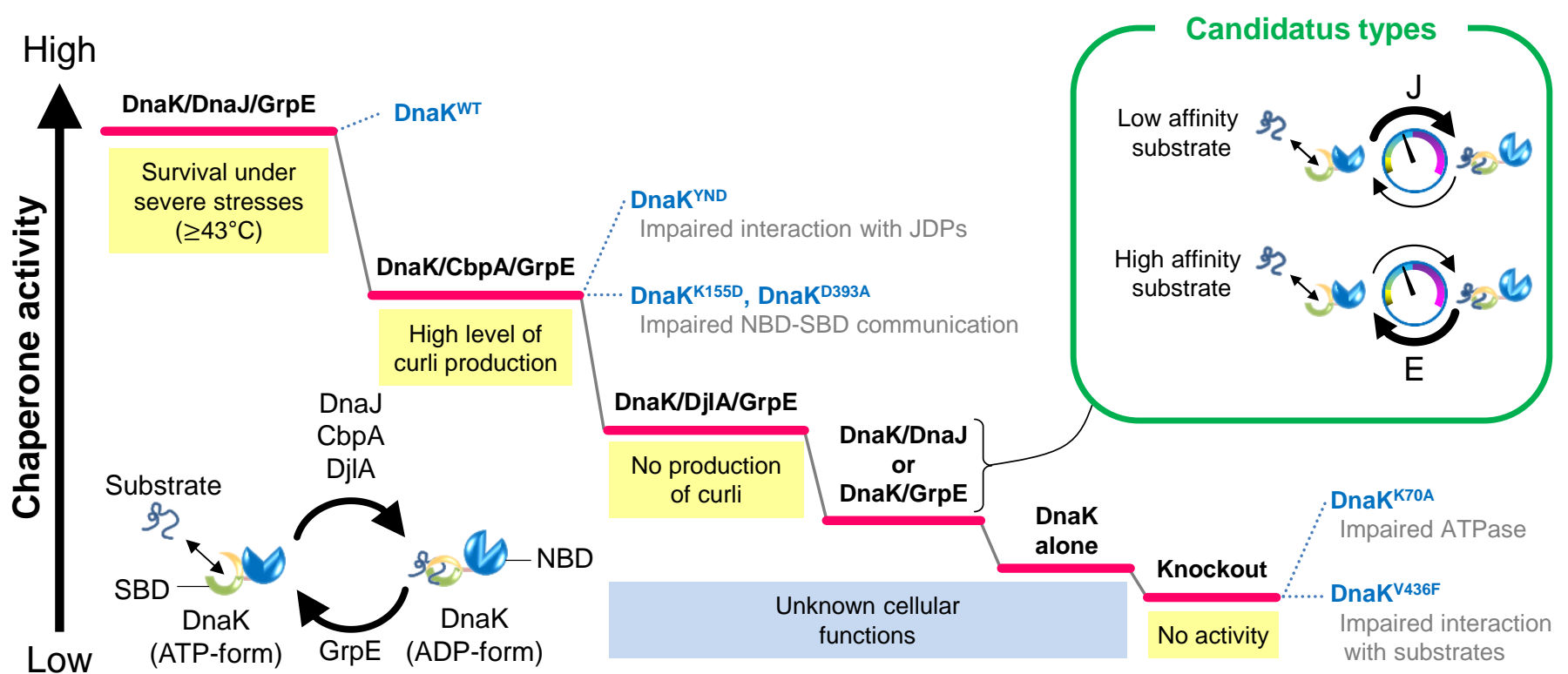